\title{
ULTRA-SONOGRAFIA ENDOSCÓPICA (USE) DA VESÍCULA BILIAR E DAS VIAS BILIARES*
}

\author{
Márcio Martins Machado ${ }^{1}$, Ana Cláudia Ferreira Rosa², Nestor de Barros³ ${ }^{3}$ Giovanni Guido \\ Cerri $^{4}$
}

\begin{abstract}
Resumo Os autores relatam as principais utilizações da ultra-sonografia endoscópica nas afecções que envolvem a vesícula biliar e as vias biliares. São descritos, ainda, alguns aspectos sonográficos à ultra-sonografia endoscópica, de condições malignas e benignas que afetam essas estruturas do aparelho digestivo, ressaltando a importância dessa metodologia no diagnóstico dessas alterações.

Unitermos: Ultra-sonografia. Ultra-sonografia endoscópica. Aparelho digestivo. Vesícula biliar. Vias biliares.
\end{abstract}

\begin{abstract}
Endoscopic ultrasonography (EUS) of the gallbladder and biliary ductal system.
The authors describe the main applications of endoscopic ultrasonography for the management of diseases of the gallbladder and biliary ductal system. In addition, some ultrasound findings of benign and malignant diseases affecting these structures of the digestive tract are presented, emphasizing the diagnostic importance of endoscopic ultrasonography.

Key words: Ultrasonography. Endoscopic ultrasonography. Digestive tract. Gallbladder. Biliary system.
\end{abstract}

\section{INTRODUÇÃO}

A ultra-sonografia endoscópica (USE) é uma modalidade recente que une as imagens da ultra-sonografia (US) convencional e os recursos visuais da endoscopia. Por meio dessa associação, juntamente com o uso de transdutores de alta frequiência, podem-se obter imagens de alta resolução. A USE permite que se estude a extensão das lesões para as adjacências do seu local de origem, auxiliando também na identificação de linfonodomegalias ${ }^{(1-11)}$.

Nesse contexto, é importante conhecermos os aspectos sonográficos das doenças estudadas por esse exame, implementando-se o diagnóstico dessas doenças.

* Trabalho realizado no Instituto de Radiologia (InRad) do Hospital das Clínicas da Faculdade de Medicina da Universidade de São Paulo (HC-FMUSP) e no Departamento de Radiologia do Hospital Sírio Libanês, São Paulo, SP.

1. Médico Radiologista, Pós-graduando do Departamento de Radiologia da FMUSP, Membro Titular do Colégio Brasileiro de Radiologia (CBR).

2. Médica Radiologista, Assistente do Departamento de Radiologia do Hospital Sírio Libanês, Pós-graduanda do Departamento de Radiologia da FMUSP, Membro Titular do CBR.

3. Professor Doutor do Departamento de Radiologia da FMUSP

4. Professor Titular do Departamento de Radiologia da FMUSP, Chefe do InRad/HC-FMUSP, Diretor da Divisão de Diagnóstico por Imagem do Instituto do Coração (InCor) do HC-FMUSP, Diretor do Departamento de Radiologia do Hospital Sírio Libanês.

Endereço para correspondência: Prof. Dr. Giovanni Guido Cerri. Instituto de Radiologia, HC-FMUSP. Avenida Dr. Enéas de Carvalho Aguiar, 255, 3ํandar. São Paulo, SP, 05403 001. E-mail: giovanni.cerri@hcnet.usp.br

Recebido para publicação em 20/8/2001. Aceito, após revisão, em 19/11/2001.

\section{VESÍCULA BILIAR}

No exame da parede da vesícula biliar pela US abdominal, o aspecto normal é representado por uma camada única ecogênica. Com a USE, utilizando-se equipamentos de freqüência de 7,5 a $12 \mathrm{MHz}$, podemos visualizar duas ou três camadas na parede vesicular normal. A correspondência dessas camadas pode ser descrita como se segue.

No caso de identificarmos três camadas, a primeira camada ecogênica representa a interface de eco marginal entre a luz da vesícula biliar e o início de sua parede. A segunda camada, hipoecogênica, representa a mucosa e a camada muscular. A terceira camada, mais ecogênica que a anterior, constitui-se na subserosa. No caso de identificarmos apenas duas camadas na parede normal da vesícula biliar, a primeira camada, hipoecogênica, representa a mucosa e a camada muscular, enquanto a segunda camada, ecogênica, representa a serosa/subserosa ${ }^{(5,6)}$.

No exame da vesícula biliar devemos sempre lembrar que ela se encontra adjacente ao bulbo duodenal, sendo que o exame de todo o órgão pode ser bastante dificultado em número significativo de pacientes. Por isso, a USE da vesícula biliar deve ser entendida como um método auxiliar à US abdominal, devendo ser analisada em conjunto com este último exame.
Na vesícula biliar, a USE auxilia especialmente no estudo detalhado do infundíbulo (colo) vesicular. Mostra-se especialmente útil na identificação de diminutos cálculos e naqueles que se encontram fixos no colo vesicular ${ }^{(7)}$. Dentre outras aplicações, podemos citar sua contribuição no diagnóstico diferencial das lesões polipóides, na avaliação do grau de invasão parietal nos tumores da vesícula biliar, na diferenciação entre lesões que se assemelham ao câncer daquelas verdadeiramente cancerosas.

O diagnóstico diferencial das lesões polipóides (Figura 1) é clinicamente importante, pois pólipos vesiculares podem ser encontrados em 6\% a $8 \%$ dos indivíduos normais durante exames de US abdominal. A maioria dos pólipos encontrados é de colesterol. Por isso, alguns autores consideram a seguinte orientação na condução desses casos: aqueles menores de $0,5 \mathrm{~cm}$ poderiam ser acompanhados com exames de US a intervalos de 12 meses; no caso de apresentarem dimensões entre 0,5 e $1,0 \mathrm{~cm}$, deveriam ser acompanhados com intervalo de seis meses; quando os pólipos apresentam mais de $1,0 \mathrm{~cm}$, existe maior probabilidade de malignidade e, provavelmente, seria indicada a colecistectomia. Ainda nessa sequiência de raciocínio, a utilização da USE forneceria informações sobre o aspecto morfológico desses pólipos e analisaria a regularidade da parede 


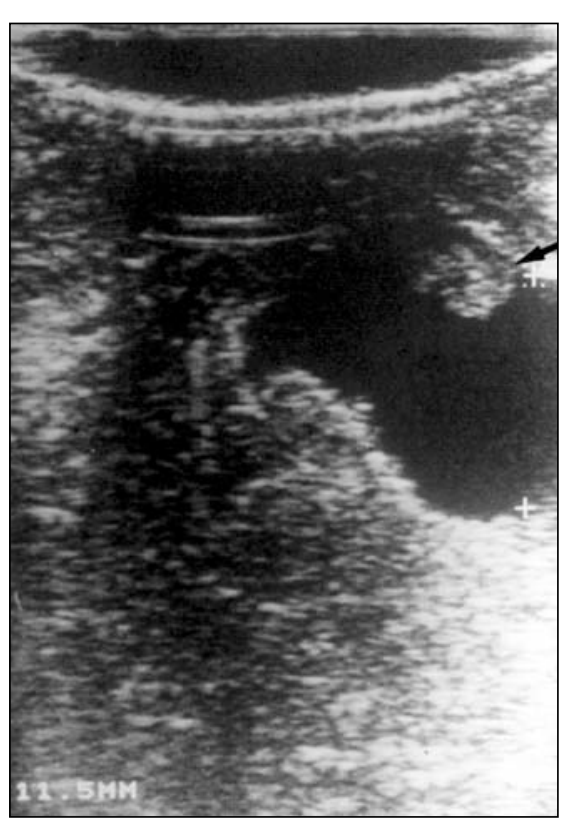

Figura 1. Pólipo na vesícula biliar (seta).

vesicular no ponto onde eles se implantam. Alguns estudos consideram que os pólipos com $0,8 \mathrm{~cm}$ ou mais poderiam ter sua avaliação complementada pelo exame de USE, auxiliando no algoritmo de estudo ${ }^{(5,6)}$.

Outras lesões, como a colecistite granulomatosa e a adenomiomatose, devem ser diferenciadas do câncer da vesícula biliar, embora muitas vezes essa diferenciação não possa ser feita. Nessas situações, poderemos encontrar lesões fúndicas (adenomiomatose segmentar), nas quais a análise cuidadosa das paredes vesiculares pela USE pode sugerir a natureza benigna ${ }^{(5,6)}$.

Como foi referido anteriormente, com a USE podemos ver a separação existente entre as camadas mucosa-muscular, da subserosa-serosa. Portanto, a USE auxiliaria na determinação de uma lesão maligna da vesícula biliar que estaria ultrapassando os limites da sua parede, pela demonstração do apagamento da estratificação habitual de suas paredes. Com relação a esta avaliação, tem sido referido que o estudo da parede vesicular livre é tecnicamente mais difícil que a avaliação da parede vesicular em contato com o leito hepático ${ }^{(5,6,8)}$. A análise cuidadosa das relações loco-regionais da vesícula biliar nos casos de tumores pode permitir a identificação de invasão da artéria hepática, assim como da veia porta. Essas informações assumem papel importante no planejamento terapêutico.
Os pólipos de colesterol apresentam, como característica à USE, o fato de serem formações arredondadas ecogênicas ou hiperecogênicas, pequenas, com reverberação sonora posterior (representando os depósitos de colesterol). Particularmente os pólipos com $0,8 \mathrm{~cm}$ ou mais tendem a apresentar aparência de formações sólidas, devendo ser diferenciados dos cânceres, dos pólipos adenomatosos e dos pólipos hiperplásicos, o que nem sempre é possível. O grande pólipo de colesterol típico apresenta, em sua superfície, pequenas irregularidades como "framboesa". Entretanto, outras lesões podem assumir este aspecto, dificultando o diagnóstico diferencial $^{(\mathbf{5 , 6 , 8})}$.

Os cânceres polipóides podem ser reconhecidos por alguns sinais. $\mathrm{O}$ aumento gradual nas dimensões dos pólipos e alterações em suas ecotexturas, observados durante o acompanhamento dessas lesões, podem indicar malignidade. Em alguns casos, com o crescimento da lesão, ocorre a diminuição de sua ecogenicidade. Aumento de diâmetro, por exemplo, de 0,8 $\mathrm{cm}$ para $1,5 \mathrm{~cm}$ (ou mais) em um ano, deve ser considerado como bastante suspeito. Embora não seja fácil, a USE de alta resolução pode sugerir também, pelo menos em alguns casos, a invasão da mucosa e muscular nas proximidades da lesão ${ }^{(\mathbf{5 , 6 , 8})}$.

$\mathrm{O}$ câncer da vesícula biliar apresenta aspectos variados, na dependência do estádio que se encontra. Ao ultra-som, podemos dividi-lo em dois tipos: aquele que se apresenta como massa e aquele que se apresenta como espessamento da parede vesicular. Em outros casos, esses tumores podem assumir o aspecto polipóide ${ }^{(\mathbf{5 , 6 , 8})}$.

Habitualmente, aqueles tumores que apresentam espessamento da parede vesicular aos exames pré-operatórios encontram-se em estádio avançado, com invasão do leito hepático. Entretanto, a USE pode ser usada nesses casos para complementar o estudo da invasão vascular e hepática $^{(5,6,8)}$.

A adenomiomatose da vesícula biliar é caracterizada, histologicamente, pelo espessamento de suas paredes e dilatação dos seios de Rokitansky-Aschoff. Por meio da USE, tanto o espessamento parietal quanto os seios de Rokitansky-Aschoff podem ser reconhecidos, sendo que estes últimos podem atingir dimensões de 1 a $2 \mathrm{~mm}$. A presença de cálculos no interior dos seios de Rokitansky-Aschoff produzem ecos posteriores, num padrão denominado de "cauda de cometa". Por conseguinte, a identificação dos seios de Rokitansky-Aschoff, juntamente com os ecos em cauda de cometa, constituem características da adenomiomatose e contribuem na diferenciação entre essas lesões e os tumores malignos da vesícula biliar ${ }^{(\mathbf{5 , 6})}$.

Uma das conseqüências de episódios pregressos de colecistite aguda é o aparecimento de lesões tumorais inflamatórias, na vesícula biliar, que devem ser diferenciadas de tumores propriamente ditos. Essas alterações, chamadas por alguns de colecistite granulomatosa, representam alterações inflamatórias da parede da vesícula biliar, podendo assumir o aspecto de imagens hipo ou hiperecogênicas. No caso das colecistites xantogranulomatosas, o aspecto hiperecogênico é bastante característico $^{(5,6)}$.

\section{VIAS BILIARES}

Como a parede dos ductos biliares é fina, ela usualmente é identificada como uma camada ecogênica, sendo algumas vezes de difícil diferenciação do tecido conjuntivo circunjacente. A USE avalia o hepatocolédoco, a porção intrapancreática do colédoco, a papila de Vater e o ducto pancreático. A porção mais alta do hepatocolédoco pode ser de difícil avaliação ${ }^{(\mathbf{5 , 6 , 8})}$.

Nas vias biliares, a USE auxilia no diagnóstico de cálculos, tumores e invasão vascular adjacente, em especial da veia porta e da artéria hepática. Nas neoplasias da papila de Vater, podemos avaliar a extensão do tumor da papila pela parede duodenal e para o parênquima pancreático, para os ductos colédoco e pancreático.

Os cálculos da via biliar principal são encontrados em aproximadamente $10 \%$ dos pacientes que apresentam colecistopatia calculosa (Figura 2). Eles se apresentam como imagens hiperecogênicas, podendo haver sombra acústica posterior associada. Mesmo pequenos cálculos têm sido identificados pela $\mathrm{USE}^{(7)}$.

No caso de tumores das vias biliares, a USE demonstra a presença de massa sólida, ora mais, ora menos ecogênica, ou 


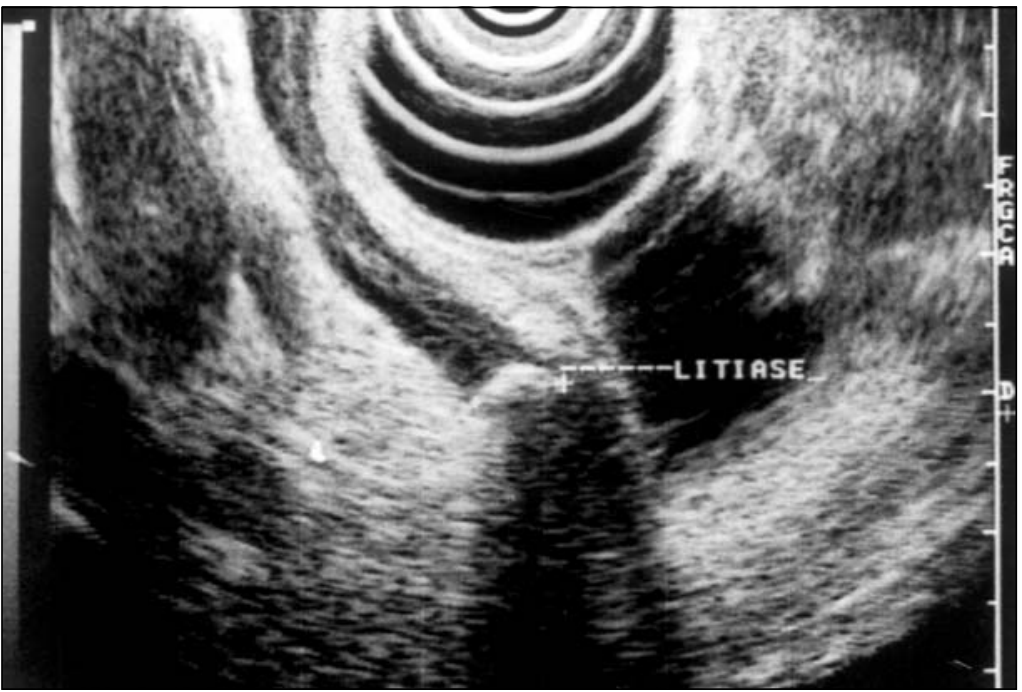

Figura 2. Coledocolitíase. Cálculo no colédoco distal.

espessamento da parede do ducto biliar. Embora seja de difícil reconhecimento na prática diária, a USE pode identificar, em algumas situações, a disseminação desses tumores pela parede do ducto hepatocolédoco. A invasão vascular é reconhecida pelo desaparecimento dos contornos ecogênicos que marcam os limites da parede

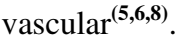

\section{PAPILA DE VATER}

Na papila de Vater, a USE participa na identificação de cálculos ou de tumores. Nos tumores, auxilia na diferenciação entre lesões pancreáticas periampulares (ade- nocarcinoma pancreático, neoplasia de colédoco distal) dos tumores verdadeiramente da papila de Vater (adenocarcinoma de papila), os quais apresentam prognóstico melhor $^{(7,9)}$.

Os cânceres da papila de Vater são identificados como massas sólidas, ora mais, ora menos ecogênicas, que crescem para o interior do colédoco distal e/ou de forma exofítica para o duodeno. A via biliar mostra-se usualmente dilatada ${ }^{(\mathbf{7 , 9})}$.

Os cálculos localizados ao nível da papila aparecem como estruturas hiperecogênicas, produtoras de sombra acústica posterior $^{(\mathbf{5 , 6 , 8})}$. Essa contribuição na diferenciação entre cálculos e tumores pode ser bastante útil no planejamento da conduta terapêutica em casos selecionados.

\section{REFERÊNCIAS}

1. Jochem RJ, Reading CC, Dozois RR, et al. Endorectal ultrasonographic staging of rectal carcinoma. Mayo Clin Proc 1990;65:1571-7.

2. Roubein LD, David C, DuBrow R, et al. Endoscopic ultrasonography in staging rectal cancer. Am J Gastroenterol 1990;85:1391-4.

3. Corman ML. Carcinoma of the rectum. In: Corman ML, ed. Colon and rectal surgery. 3rd ed. Philadelphia, PA: JB Lippincott, 1993:596-720.

4. Torres GM. Endoscopic ultrasonography. In: Gore RM, Levine MS, Laufer I, eds. Textbook of gastrointestinal radiology. 1st ed. Philalelphia, PA. WB Saunders, 1994:127-39.

5. Yasuda K, Nakajima M, Kawai K. Technical aspects of endoscopic ultrasonography of the biliary system. Scand J Gastroenterol Suppl 1986; 123:143-6.

6. Mitake M, Nakazawa S, Naitoh Y, et al. Endoscopic ultrasonography in diagnosis of the extent of gallbladder carcinoma. Gastrointest Endosc 1990;36:562-6.

7. Ganc AJ, Ardengh J. Ultra-sonografia endoscópica em gastroenterologia. In: Mincis M, ed. Gastroenterologia e hepatologia. Diagnóstico e tratamento. São Paulo, SP: Lemos Editorial, 1998:65-80.

8. Mukai H, Nakajima M, Yasuda K, Mizuno S, Kawai K. Evaluation of endoscopic ultrasonography in the pre-operative staging of carcinoma of the ampulla of Vater and common bile duct. Gastrointest Endosc 1992;38:676-83.

9. Maluf Filho F. Contribuição da ecoendoscopia à avaliação das neoplasias malignas do pâncreas e da papila duodenal maior. [Tese de Doutorado]. São Paulo: Universidade de São Paulo, 2000.

10. Boyce GA, Sivak MV Jr. Endoscopic ultrasonography in the diagnosis of pancreatic tumors. Gastrointest Endosc 1990;36(2 Suppl):S28-32.

11. Fein J, Gerdes H. Localization of islet cell tumors by endoscopic ultrasonography. Gastroenterology 1992;103:711-5. 